\title{
Beta human chorionic gonadotropin concentrations in serum of patients with pancreatic adenocarcinoma
}

K N Syrigos, I Fyssas, M M Konstandoulakis, K J Harrington, S Papadopoulos, N Milingos, P Peveretos, B C Golematis

\begin{abstract}
Background-Human chorionic gonadotropin (hCG) is normally produced and secreted by trophoblastic cells during pregnancy and from gestational trophoblastic neoplasms. It is also detected in ovarian, stomach, and colon adenocarcinomas, as well as in squamous cell carcinoma of the oesophagus. Recently, interest in its role in the pathogenesis of tumours has been enlivened after the presence of $\beta$ hCG in the cell membrane of several malignant cells was shown in vitro. Aims-To investigate the circulating concentrations of $\beta \mathrm{hCG}$ in patients with exocrine pancreatic adenocarcinoma and to examine its potential prognostic value. Patients-Thirty six patients with exocrine pancreatic adenocarcinoma, 12 patients with chronic pancreatitis, and 21 healthy volunteers were studied.

Methods-BhCG serum concentrations were detected by the application of a radioimmunoassay technique.

Results-Fifteen of 36 patients with pancreatic adenocarcinoma and only one patient with chronic pancreatitis had detectable plasma concentrations of $\beta$ hCG $(p<0.01)$. The patients with circulating serum titres of $\beta$ hCG had a worse outcome compared with the group of $\beta$ hCG negative patients: the difference was statistically significant $(p=0.01)$.

Conclusion-More than $40 \%$ of pancreatic exocrine tumours produce $\beta$ hCG and its production is correlated with an adverse effect on outcome.

(Gut 1998;42:88-91)
\end{abstract}

Keywords: $\beta$-human chorionic gonadotropin; chorionic gonadotropin; pancreatic cancer; tumour marker; paraneoplastic syndrome

Adenocarcinoma of the pancreas is the fifth leading cause of cancer in Western countries and an almost uniformly fatal disease. ${ }^{1}$ Early diagnosis of non-metastatic cancer, however, may result in a more favourable outlook. ${ }^{2}$ Unfortunately, despite advances in diagnostic imaging, most pancreatic cancers continue to be recognised at a late stage, ${ }^{34}$ and screening asymptomatic persons with serum tests and/or ultrasound has been disappointing. ${ }^{56}$ Such a screening programme would be cost effective only in a cohort with a high probability of pancreatic cancer. A biological parameter with diagnostic value for pancreatic adenocarcinoma is therefore required.

Cancer patients often present with signs and symptoms not directly related to the malignant lesions. These symptoms may be due to paraneoplastic syndromes caused by biologically active proteins produced by the tumour cells, including growth factors and hormones, such as $\beta$-human chorionic gonadotropin ( $\beta$ hCG).

Human chorionic gonadotropin is a glycoprotein hormone normally produced and secreted by trophoblastic cells during pregnancy and also from tumours called gestational trophoblastic neoplasms, arising from those cells. As this hormone is, in general, not detectable in the serum of healthy nonpregnant individuals, ${ }^{7} \beta \mathrm{hCG}$ is considered to be of malignant origin and has diagnostic value in the detection and follow up of several neoplasms. ${ }^{78}$ In addition to tumours of trophoblastic origin, malignancies in which $\beta$ hCG is detected include ovarian, stomach, and colon adenocarcinomas, as well as squamous cell carcinoma of the oesophagus. ${ }^{910}$ Recently, there has been renewed interest in the role of $\beta h C G$ in the pathogenesis of tumours ${ }^{11}$ following the demonstration of the presence of $\beta$ hCG in the cell membrane of several malignant cells in vitro. ${ }^{12} 13$

The objective of the present study was to investigate the circulating concentrations of $\beta$ hCG in patients with exocrine pancreatic adenocarcinoma and to compare them with circulating concentrations of the same hormone in healthy individuals and in patients with chronic pancreatitis. We also examined the potential prognostic value of this biological marker in those patients with pancreatic neoplastic disease. Our study, as far as we know, is the first of this kind.

Patients, materials, and methods STUDY GROUPS

Pancreatic adenocarcinoma

Thirty six patients (19 men and 17 women, mean (range) age 63.8 (40-82) years) with newly diagnosed and histologically confirmed (after an open biopsy) exocrine pancreatic adenocarcinoma were studied. All patients were randomly selected and the malignant tumours were classified and graded using established histopathological and surgical staging systems based on the TNM classification, ${ }^{14-16}$ as follows: stage I, two patients; stage II, three patients; stage III, 11 patients; and stage IV, 20 patients. All patients underwent laparotomy aiming at 
achieving either radical resection of the tumour, or palliative surgical bypass. However, radical resection was not possible in any of the patients in this study. In four patients a debulking procedure was performed, but the histological margins of the resected specimen were not free of disease. In eight patients, following intraoperative assessment that the disease was unresectable, yet not threatening to cause obstructive sequelae, no further surgical procedure was undertaken. In the remaining 24 patients a bypass procedure alone was performed: gastrojejunostomy $(5 / 24,21 \%)$, cholecystojejunostomy and choledochojejunostomy $(5 / 24,21 \%)$, combination of gastrojejunotomy and either cholecystojejunostomy or choledochojejunostomy $(11 / 24,46 \%)$, cholocystostomy $(2 / 24,8 \%)$, and intraoperative cauterisation with the use of a microwave source $(1 / 24$, $4 \%$ ). The surgical procedures performed on patients with stage I/II disease were as follows: gastrojejunostomy in two patients, cholecystojejunostomy and choledochojejunostomy in one patient, and no surgical procedure in two patients. These procedures match those undergone by the group of patients with stage III/IV disease. Following recovery from surgery, 21 patients ( $3 / 5$ stage I/II and $18 / 31$ stage III/IV) received palliative chemotherapy using a 5-fluorouracil and folinic acid schedule. Twenty eight patients received palliative radiotherapy to symptomatic bone metastases. Radiotherapy was not administered to the primary pancreatic tumour.

\section{Chronic pancreatitis}

Twelve patients with chronic pancreatitis (six men and six women, mean (range) age 62.5 (42-79) years) were studied.

\section{Healthy volunteers}

Twenty one apparently healthy volunteers were used as an age matched negative control group (12 men and nine women, mean age 63.5 years).

All participants had given their informed written consent before entering the study. Blood samples were drawn between 08.00 and 10.00 hours; plasma was separated, frozen, and blindly analysed. None of the participants had previously undergone any kind of anticancer treatment (surgery, chemotherapy, or radiotherapy), nor had testicular disease, as confirmed by clinical and ultrasonographic examination. All female participants were postmenopausal.

Measurements for plasma $\beta$ hCG concentrations were performed in duplicate with the use of the commercially available kit from Peninsula (USA) and the application of a radioimmunoassay (RIA) technique. The method has a lower sensitivity limit of $2.5 \mathrm{mIU} / \mathrm{ml}$ and does not cross react with luteinising hormone.

STATISTICAL ANALYSIS

For the evaluation of the presence of serum $\beta$ hCG between the groups studied, Yates's corrected $\chi^{2}$ test was used. For the evaluation of the survival differences between the subgroups of $\beta$ hCG positive and negative pancreatic

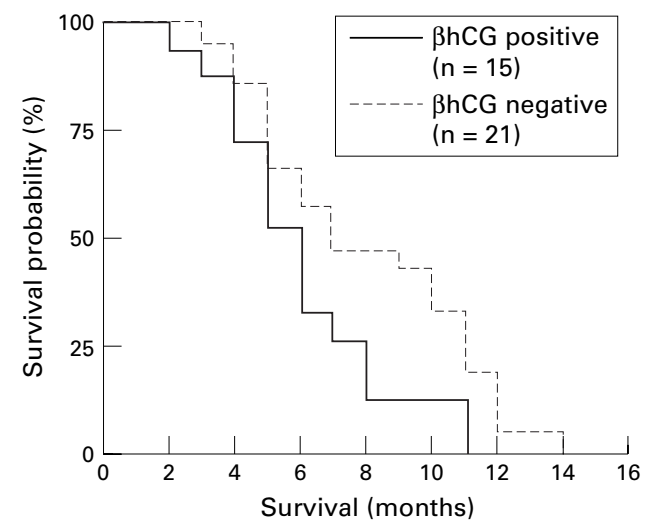

Figure 1 Survival curves for patients with pancreatic adenocarcinoma relative to $\beta h C G$ status $(p<0.01)$.

adenocarcinoma patients, the survival curves were estimated by the Kaplan-Meier product limit method and compared by the log rank test, using STATA software.

\section{Results}

As $\beta$ hCG is not found in healthy, non-pregnant individuals, we considered all serum samples with detectable $\beta$ hCG, of any concentration, as abnormal. Fifteen of $36(42 \%)$ patients with pancreatic adenocarcinoma had detectable $\beta$ hCG plasma concentrations. These consisted of 5/19 men $(40 \%)$ and $10 / 17$ women $(59 \%)$. The difference in incidence of detectable BhCG between male and female pancreatic cancer patients was found to be statistically significant $(p<0.05)$. Only one $(8 \%)$ patient with chronic pancreatitis had detectable $\beta$ hCG plasma concentrations. None of the normal individuals had detectable plasma titres of $\beta$ hCG. The difference between the group of patients with malignant disease and the two control groups was statistically significant $(\mathrm{p}<0.05$ and $\mathrm{p}<0.01$ respectively)

The prevalence of detectable $\beta$ hCG concentrations among pancreatic adenocarcinoma patients at different stages was as follows: stage I, 0 patients; stage II, $1 / 3$ patients; stage III, $3 / 11$ patients; and stage IV, $11 / 20$ patients. No statistically significant difference was found in the prevalence of detectable $\beta$ hCG concentrations when patients of stages I and II were compared with patients of stages III and IV $\left(\chi^{2}=0.32\right)$. The patients with detectable serum titres of $\beta$ hCG had a worse outcome compared with the group of $\beta$ hCG negative patients: the difference was statistically significant $(p=0.01)$. Figure 1 illustrates overall survival of these two groups relative to the detection of $\beta$ hCG.

\section{Discussion}

With recent advances in the management of early pancreatic cancer, it is now appropriate to focus efforts on earlier diagnosis. Unfortunately this is not easy because of the non-specific nature of the early symptoms and the very large numbers of people who would need to be screened. After decades of investigation, a simple laboratory test that could identify the presence of an otherwise undetectable tumour remains an elusive goal. Several molecular and biological markers have 
been suggested as having diagnostic value in pancreatic cancer, the most reliable of which is CA 19-9. Nevertheless, further studies are needed to provide a better understanding of the pathogenesis of pancreatic adenocarcinoma, which could ultimately lead to more effective detection and treatment.

Zondek was the first to suggest, in 1929, the value of hCG determination in the diagnosis of trophoblastic tumours. The development of an RIA method, with high sensitivity and specificity for $\beta$ hCG, made this marker essential in the diagnosis, screening, and monitoring of choriocarcinomas. Nevertheless, despite concerted research efforts, the clinical application of $\beta$ hCG as a cancer marker in other malignancies has been sporadic. ${ }^{17}$ Several researchers have reported a low incidence $(8-20 \%)$ of detectable plasma $\beta$ hCG in patients with malignant tumours of the gastrointestinal (GI) tract. ${ }^{101819}$ Others have shown the presence of $\beta$ hCG in malignant cells of the GI tract with the use of immunohistochemical techniques. ${ }^{20}$ It has also been shown that hCG positive cells in colon adenocarcinoma were arranged in syncytial clumps or columns resembling trophoblastic tissue. ${ }^{21}{ }^{22} \mathrm{~A}$ further proof of the ectopic production of hCG by malignant cells was provided by the in vitro work of Tashjian $e t a l^{23}$ and Rosen et $a l^{24}$ in carcinoma cell lines.

In our study, the prevalence of pancreatic cancer patients with detectable serum concentrations of $\beta$ hCG was found to be as high as $42 \%$. This rate is higher than previously reported in other non-gestational trophoblastic neoplasms. ${ }^{101825}$ We also found a higher incidence of $\beta \mathrm{hCG}$ positive women than men ( $59 \%$ versus $40 \%$ ). This discrepancy between the sexes has been reported for other hormones in patients with pancreatic adenocarcinoma ${ }^{26} 27$ and it may be indicative of hormonal involvement in the pathogenesis of the disease.

According to our findings we were not able to establish a relationship between detectable $\beta$ hCG serum concentrations and the clinical stage of the disease. This observation is in accordance with previous findings in other tumours, such as colonic and gastric adenocarcinomas, and may be explained by the fact that each pancreatic tumour either produces $\beta$ hCG or not, regardless of the tumour bulk. ${ }^{10}$ However, the small number of patients with early stage (I and II) disease has hampered our ability to examine this matter in more detail. In those patients with detectable $\beta$ hCG concentrations, we were able to show a more aggressive clinical course and poorer prognosis. These results are in agreement with an earlier report suggesting that $\beta$ hCG is found more commonly in patients with advanced colonic adenocarcinoma. ${ }^{20} \mathrm{~A}$ possible explanation of this phenomenon could be the significant immunosuppressive effect of $\beta$ hCG. ${ }^{28}$ The biological activity of the highly sialyted small glycoproteins, including hCG (the molecular weight of hCG is $37 \mathrm{kDa}$ and $30 \%$ of it is sugar) has been extensively investigated in the past. It seems that these molecules may play a role in reducing immune responsiveness against the fetoplacental unit and malignancies. ${ }^{29-32}$ Another possible explanation could be that the tumours secreting $\beta$ hCG have undergone retrodifferentiation towards more anaplastic forms through metaplastic proliferation. It has already been clinically shown that $\beta$ hCG expression is indicative of intense tumour growth, metastatic aggressiveness, and poor therapeutic response in many malignancies, including bladder ${ }^{33}$ and vulvovaginal ${ }^{34}$ cancer. It seems that the expression of hCG genes by malignant cells is of great clinical importance, because it allows the malignant cells to regulate their growth independently, since $\beta$ hCG has been shown to have properties of a growth factor. $^{35}$ The correlation of circulating $\beta$ hCG with poorer outcome should be seen in light of the knowledge that our patients received various treatments, as previously described. Since none of these patients underwent a radical resection and previous studies have shown that palliative bypass procedures do not improve the survival of patients with pancreatic adenocarcinoma, ${ }^{3637}$ it is unlikely that the differences in survival between patients with $\beta \mathrm{HCG}$ positive and $\beta H C G$ negative tumours are due to differences in the extent of surgical treatment. Indeed, there were no systematic differences in the surgical procedures undertaken in the two groups of patients, those with detectable and those with undetectable serum concentrations of $\beta \mathrm{HCG}$. Similarly, the impact of palliative chemotherapy and radiotherapy are unlikely to account for these data.

As expected, none of the healthy individuals ${ }^{24}$ and only one of the patients with chronic pancreatitis had detectable $\beta$ hCG serum concentrations. Similar observations have been made occasionally in the past, for a few patients with non-malignant disorders of the GI tract, including regional enteritis, ulcerative colitis, and gastric and duodenal ulcer. ${ }^{38}$ It is of particular interest that some of these conditions are associated with precancerous lesions and malignant transformation, indicating a possible involvement of $\beta \mathrm{hCG}$ in the pathogenesis of neoplastic disease.

Our results provide further support for the "trophoblastic theory of cancer", initially presented by Professor John Beard in 1902 and recently reviewed by Gurchot. ${ }^{39}$ They also provide further scientific basis for the use of hCG in the prevention, diagnosis, and treatment of cancer. As phase I clinical trials of vaccines against hCG in patients with cancer have been completed, pancreatic cancer patients should be considered as potential candidates for the phase II trials that will be initiated shortly. ${ }^{40}$

In conclusion, in the present study we have shown that over $40 \%$ of the patients with pancreatic cancer had detectable serum concentrations of $\beta \mathrm{hCG}$ and that these patients had a worse prognosis. Further studies are needed to explain the role of $\beta$ hCG in the development and pathogenesis of pancreatic adenocarcinoma.

1 National Cancer Institute. Annual Cancer Statistics Review 1990:1973-87.

2 Nix GA, Schmitz PJ, Wilson JHP. Carcinoma of the head of the pancreas. Therapeutic implications of endoscopic retrograde pancreatography. Gastroenterology 1984;87:
$37-43$. 
3 Savarino V, Mansi C, Bistolfi L. Failure of new diagnostic aids in improving detection of pancreatic cancer at a aids in improving detection of pancreatic
resectable stage. Dig Dis Sci $1983 ; 28: 1078-82$.

4 Cubilla AL, Fitzgerald PJ. Pancreas cancer (nonendocrine). A review. Part I. Clinical Bulletin of the Memoria Sloan-Kettering Cancer Center 1994;8:91-9.

5 Moosa AR, Levin B. The diagnosis of early pancreatic cancer: the University of Chicago experience. Cancer 1981 47:1688-92.

6 Di Magno EP. Early diagnosis of chronic pancreatitis and pancreatic cancer. Med Clin North Am 1988,72:979-92.

7 Rosen SW, Weintraub BD, Aaronson SA. Non-random ectopic protein production by malignant cells: direct evidence in vitro. 7 Clin Endocrinol Metab 1980;50:834-41.

8 Braunstein GD, Vaitukaitis JL, Carbone PP, et al. Ectopic production of hCG by neoplasms. Ann Intern Med 1973;78:39-45.

9 Papapetrou PD, Sakarelou NP, Braouzi H, et al. Ectopic production of hCG in malignant tumors. Cancer 1978;42. production

10 Birkenfeld S, Noiman G, Krispin M, et al. The incidence and significance of serum hCG and CEA in patients with gastrointestinal of 1989;15:103-8

11 Regelson W. Have we found the "definitive cancer biomarker"? Cancer 1995;76:1299-301.

12 Acedevo HF, Tong JY, Hartsock RJ. Human chorionic gonadotropin-beta subunit gene expression in cultured human fetal and cancer cells of different types and origins. Cancer 1995;76:1467-75.

13 Acedevo HF, Krichevsky A, Campbell-Acevedo EA, et al. Expression of membrane associated human chorionic gonadotropin, its subunits and fragments by cultured human cancer cells. Cancer 1992;69:1829-42.

14 Cancer of the Pancreas Task Force. Staging of cancer of the pancreas. Cancer 1981;47:163-9.

15 Hermanek P, Sobin LH. UICC, TNM classification of malignant tumours. Berlin: Springer Verlag, 1987:65-7.

16 Kloppel G, Maillet B. Classification and staging of pancreKloppel G, Maillet B. Classification and staging of pancreatic nonen-12.

17 Bagshawe KD. Choriocarcinoma: a model for tumor markers. Acta Oncol 1992;31:99-106.

18 Gailani S, Ming Chu T, Nussbaum A, et al. hCG in nontrophoblastic neoplasms. Cancer 1976;38:1684-6.

19 Hattori M, Fukase M, Yoshim H, et al. Ectopic production of hCG by neoplasms. Cancer 1978;42:2328-33.

20 Buckely CH, Fox H. An immunohistochemical study of the significance of hCG secretion by large bowel adenocarcinoma. F Clin Pathol 1979;32:368-72.

21 Shousha S, Chappell R, Mattews J, et al. Human chorionic gonadotropin expression in colorectal adenocarcinoma. Dis Colon Rectum 1987;29:558-60.

22 Metz RA, Ritcher JJ, Leder LD. Adenocarcinoma of colon with syncytiotrophoblastic differentiation: differential diag-

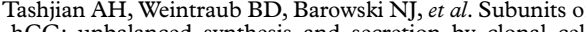
hCG; unbalanced synthesis and secretion by clonal cell Acad Sci USA 1975;70:1419-22.
24 Rosen SW, Weintraub BD, Vaitukaitis JL, et al. Placental proteins and their subunits as tumor markers. Ann Intern Med 1975;82:71-83.

25 Muggia FM, Rosen SW, Weintraub BD, et al. Ectopic placental proteins in nontrophoblastic tumors: serial measurements following chemotherapy. Cancer 1975;36:132737.

26 Greenway BA, Iqbal MJ, Johnson PI, et al. Low serum testosterone concentrations in patients with carcinoma of the pancreas. BMF 1983;286:93-4.

27 Militello C, Sperti C, Foreita C, et al. Plasma levels of testosterone and hypophyseal gonadotropins in men affected by pancreatic cancer. Digestion 1984;30:106-11.

28 Adcock EW, Teasdale T, August CS, et al. Human chorionic gonadotropin: its possible role in maternal lymphocyte suppression. Science 1973;181:845-7.

29 Starkey PM. Natural killer cells/large granular lymphocytes in pregnancy. Introduction. In: Lewis CE, McGee JO'D, eds. The natural killer cell. New York: Oxford University Press, 1992:206-8.

30 Van Rinsum J, Smets LA, Van Rooy H, et al. Specific inhibition of human natural killer cell-mediated cytotoxicity by sialic acid and sialo-oligosaccharides. Int 7 Cancer 1986;38 sialic acid 22 .

31 Acedevo HF, Kellen JA, Wong ACH, et al. Expression of human choriogonadotropin-like material correlates with metastatic phenotype of R3230 AC rat adenocarcinoma. Cancer Invest 1987;5:177-85.

32 Hanna N, Filder I. Relationship between metastatic potential and resistance to natural killer cell-mediated cytotoxicity in three murine tumor systems. 7 Natl Cancer Inst 1981; 6:1183-90.

33 Moutzoauris G, Yannopoulos D, Barbatis C, et al. Is $\beta$ hCG production by transitional cell carcinoma of the bladder a marker for aggressive disease and resistance to radiomarker for aggressive disease and
therapy? Br f Urol 1993; 72:807-9.

34 Carter PG, Iles RK, Neven P, et al. Measurement of urinary beta core fragment of human chorionic gonadotropin in women with vulvovaginal malignancy and its prognostic significance. Br f Cancer 1995;71:350-3.

35 Melmed S, Braunstein GD. Human chorionic gonadotropin stimulates proliferation of $\mathrm{Nb} 2$ rat lymphoma cells. 7 Clin Endocrinol Metab 1983;56:1068-70.

36 Wade TP, Radford DM, Virgo KS, et al. Complications and outcomes in the treatment of pancreatic adenocarcinoma in the United States Veteran. F Am Coll Surg 1994;179:38-

37 Watanapa P, Williamson RCN. Surgical palliation for pancreatic cancer: development during the past two decades. Br f Surg 1992;79:8-20.

38 Vaitukaitis JL, Rosen SW, Weintraub BD, et al. Gonadotropins and their subunits: basic and clinical studies. Recent Prog Horm Res 1976;32:289-331.

39 Gurchot C. The trophoblast theory of cancer. Oncology 1975;31:310-33.

40 Triozzi PL, Gochnour D, Martin EW, et al. Clinical and immunological effects of a synthetic $\beta$-human chorionic gonadotropin vaccine. Int f Oncol 1994;5:1447-53. 